$\underline{\xi}=\mathbf{z}$

\title{
Progression of bifurcated family f type periodic orbits in the circular restricted three-body problem
}

\author{
Nishanth Pushparaj ${ }^{1 @}$, Ram Krishan Sharma ${ }^{1 *}$ \\ ${ }^{1}$ Department of Aerospace Engineering, Karunya University, Coimbatore - 641114, Tamilnadu, India \\ @ Present address: Division of Mechatronics, Madras Institute of Technology, Anna University, Chennai - 600044, Tamil Nadu, India \\ *Corresponding author E-mail: ramkrishan@karunya.edu
}

\begin{abstract}
Progression of f-type family of periodic orbits, their nature, stability and location nearer the smaller primary for different mass ratios in the framework of circular restricted three-body problem is studied using Poincaré surfaces of section. The orbits around the smaller primary are found to decrease in size with increase in Jacobian Constant C, and move very close towards the smaller primary. The orbit bifurcates into two orbits with the increase in $\mathrm{C}$ to 4.2. The two orbits that appear for this value of $\mathrm{C}$ belong to two adjacent separate families: one as direct orbit belonging to family g of periodic orbits and other one as retrograde orbit belonging to family f of periodic orbits. This bifurcation is interesting. These orbits increase in size with increase in mass ratio. The elliptic orbits found within the mass ratio $0<\mu \leq 0.1$ have eccentricity less than 0.2 and the orbits found above the mass ratio $\mu>0.1$ are elliptical orbits with eccentricity above 0.2. Deviations in the parameters: eccentricity, semi-major axis and time period of these orbits with solar radiation pressure $\mathrm{q}$ are computed in the frame work of photogravitational restricted Three-body problem in addition to the restricted three-body problem. These parameters are found to decrease with increase in the solar radiation pressure.
\end{abstract}

Keywords: Restricted Three-Body Problem; Poincaré Surfaces of Section; Solar Radiation Pressure; Sun-Jupiter System; Direct and Retrograde Periodic Orbit.

\section{Introduction}

Periodic orbits in planar circular restricted three-body problem (RTBP) play an important role in understanding the predominant properties of dynamical systems and have been very extensively studied in celestial mechanics. Szebehely [23] has an extended record of qualitative studies on restricted three-body problem. Poincaré surfaces of section (PSS) method is one of the productive methods which helps in computing periodicity, stability, ergodicity characteristics of a trajectory [13]. There have been numerous studies directed at finding periodic and quasi-periodic orbits around the liberation points using PSS method. Some of the illustrious works on periodic, quasi-periodic and chaotic orbits and in locating stability regions in the RTBP were done by Broucke [3], Henon [9], Jefferys [10], Bruno [4], Smith \& Szebehely [23]. Winter \& Murray [27], [28], [29], [30] used this technique to explore the phase space of Sun-Jupiter system and to study the resonance and chaos of the system. Sharma \& Subba Rao [21], [22], Subba Rao \& Sharma [24] and Sharma [18], [19], [20] have studied the perturbation effects on the Lagrangian points in the RTBP. Dutt \& Sharma [6] determined the location of the periodic orbits and their stability in terms of maximum amplitude of oscillation. Dutt \& Sharma [7] studied the evolution of periodic orbits in SunMars system using PSS method in the framework of Photogravitational Restricted Three- Body Problem (PRTBP). Further, Beevi \& Sharma [1] analysed the periodic orbits around Saturn-Titan system. Later, they continued the study by considering Saturn's oblateness effect on the periodic orbits of that system [2]. Recently, Pushparaj \& Sharma [15] studied the interior resonance periodic orbits in Sun-Jupiter system.
The present work explores the location, nature and size of the periodic orbits in the framework of RTBP near smaller primary in Sun-Jupiter system using PSS method, whose mass ratio is $\mu=$ 0.0009537284 [21]. Further, the study has been carried out for different mass ratios for certain values of Jacobi constant $\mathrm{C}$ to study the location, nature and size of these periodic orbits of family f bifurcated from family g of periodic orbits in the RTBP [3], [4], [5]. Deviations in the parameters: eccentricity, semi-major axis and time period of these two families of periodic orbits with the effect of solar radiation pressure are also computed. These parameters are found to decrease with the increase in solar radiation pressure.

\section{Equations of motion}

The radiation force on a particle, exerted by a radiating body, generally consists of three terms, namely the radiation pressure, the Doppler shift of the incident radiation and the Poynting drag (Poynting [14], Robertson [17]). The first two act radially and the third one acts opposite to the velocity vector. The latter two components are caused by absorption and subsequent reemission of radiation and constitute Poynting-Robertson effect. Radzievskii [16] pointed out that this effect is negligible and that the only significant force is radiation pressure. After that Radzievskii's model has been utilized in a large number of studies.

The effect of radiation pressure of a source can be expressed by a mass reduction factor $\mathrm{q}=1-\varepsilon$, where the radiation coefficient $\varepsilon$, is the ratio of the force $F_{p}$ which is caused by radiation to the force $\mathrm{F}_{\mathrm{g}}$ which results from gravitation, that is $\varepsilon=\mathrm{F}_{\mathrm{p}} / \mathrm{F}_{\mathrm{g}}$, $\mathrm{q}$ is expressed 
in terms of particle radius ' $a$ ', density ' $\delta$ ' and radiation pressure efficiency ' $\chi$ ' (in cgs system).

$\mathrm{q}=1-\frac{5.6 \times 10^{-5}}{\mathrm{a} \delta} \chi$.

Knowing the mass and the luminosity of the radiating body, $\varepsilon$ can be found for any given radius and density. Solar radiation pressure force $F_{p}$ changes with distance by the same law of gravitational attraction force $F_{g}$ and acts opposite to it. Thus, the Sun's resulting force acting on the particle is (Sharma [20]; Kalvouridis et al. [11])

$F=F_{g}-F_{p}=\left(1-\frac{F_{p}}{F_{g}}\right) F_{g}=F_{p}(q)$.

If $\mathrm{q}=1$, the radiation pressure has no effect. If $\mathrm{q}<0$, then radiation surpasses gravity and if $0<\mathrm{q} \leq 1$, gravitational force exceeds radiation.

In the dimensionless synodic coordinate system Oxy with origin of the system positioned on the centre of mass of the primaries, the more massive and smaller primary lie on the Ox-axis at $(-\mu, 0)$ and $(1-\mu, 0)$, respectively. The equations of motion of the third body are (Sharma [20])

$\ddot{\mathrm{x}}-2 \dot{y}=\frac{\partial \Omega}{\partial x}$,

$\ddot{\mathrm{y}}+2 \dot{x}=\frac{\partial \Omega}{\partial y}$,

where

$\Omega=\frac{1}{2}\left(\mathrm{x}^{2}+\mathrm{y}^{2}\right)+\frac{\mathrm{q}(1-\mu)}{\mathrm{r}_{1}}+\frac{\mu}{\mathrm{r}_{2}}$,

$\mathrm{r}_{1}^{2}=(x+\mu)^{2}+y^{2}, \mathrm{r}_{2}^{2}=(x-1+\mu)^{2}+y^{2}$.

The Jacobi integral is given by

$\dot{\mathrm{x}}^{2}+\dot{y}^{2}=2 \Omega-C$.

\section{Poincaré Surfaces of Section and stability of the periodic orbits}

PSS is a widely used technique in locating the periodic, quasiperiodic and chaotic orbits. To determine the orbital elements of the test particle at any instant it is necessary to know its initial position $(\mathrm{x}, \mathrm{y})$ and velocity $(\dot{\mathrm{x}}, \dot{\mathrm{y}})$, which corresponds to a point in a four-dimensional phase space. We have constructed surfaces of sections in the $\mathrm{x}, \dot{\mathrm{x}}$ plane. The initial values were selected along the Ox-axis by using intervals of length between 0.0001 and 0.01 .The magnitude of velocity vector was determined from Jacobian constant $\mathrm{C}$ which is a function of the velocity vector. Moreover, the fine discretization of position along the $\mathrm{x}$-axis guarantees an extensive coverage of phase plane since each trajectory, regardless of the complexity of its motion, has a unique path through the phase plane. By defining the plane, say $y=0$, resulting in threedimensional space, the values of $\mathrm{x}$ and $\dot{\mathrm{x}}$ can be plotted every time the particle has $\mathrm{y}=0$, whenever trajectory intersects the plane in a particular direction, say $\dot{y}>0$.

Kolmogorov-Arnold-Moser (KAM) theory provides the stability condition for the periodic orbits in the planar restricted three-body problem. PSS are used to know the regular and chaotic behavior of a trajectory. For a regular trajectory, there exists a stable region of islands in the PSS or a curve shrinks to a point then there is existence of a periodic orbit. Any irregular distribution of points on PSS describes that the trajectory is chaotic in behavior. In general, regular regions of PSS are defined by periodic orbits and surrounded by an area of quasi-periodic orbits. The regular regions can be interpreted as regions of stability in the sense that outside them the motion is unstable or chaotic in nature and inside them the motion is regular.

\section{Periodic orbit around Jupiter in Sun- Jupiter system}

The initial conditions for numerical integration are chosen for a constant solar radiation pressure and different values of C. Fourthorder fixed step size Runge-Kutta-Gill method is used to integrate the equations of motion (1) and (2) to generate the PSS. With the same value of $C$, the distance interval $(\Delta x)$ for each of the two starting conditions is provided as input and integration has been performed for different values of $\mu$ without considering any perturbations such as oblateness or radiation pressure. In the case of Sun-Jupiter system, we have generated the PSS for the mass ratio $\mu=0.0009537284$ with different values of C (3.0 to 4.0). The periodic orbits around Jupiter located from PSS are shown in Figs.1a to 1d.

(A)

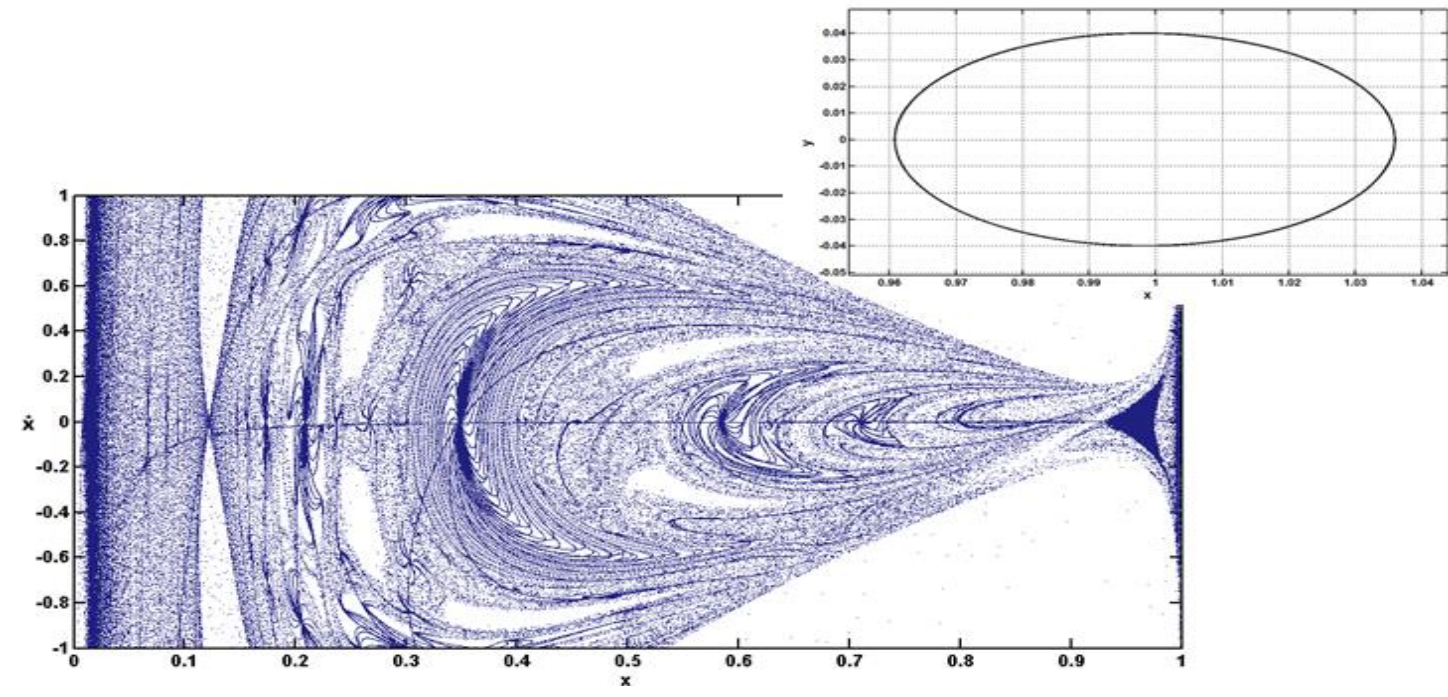




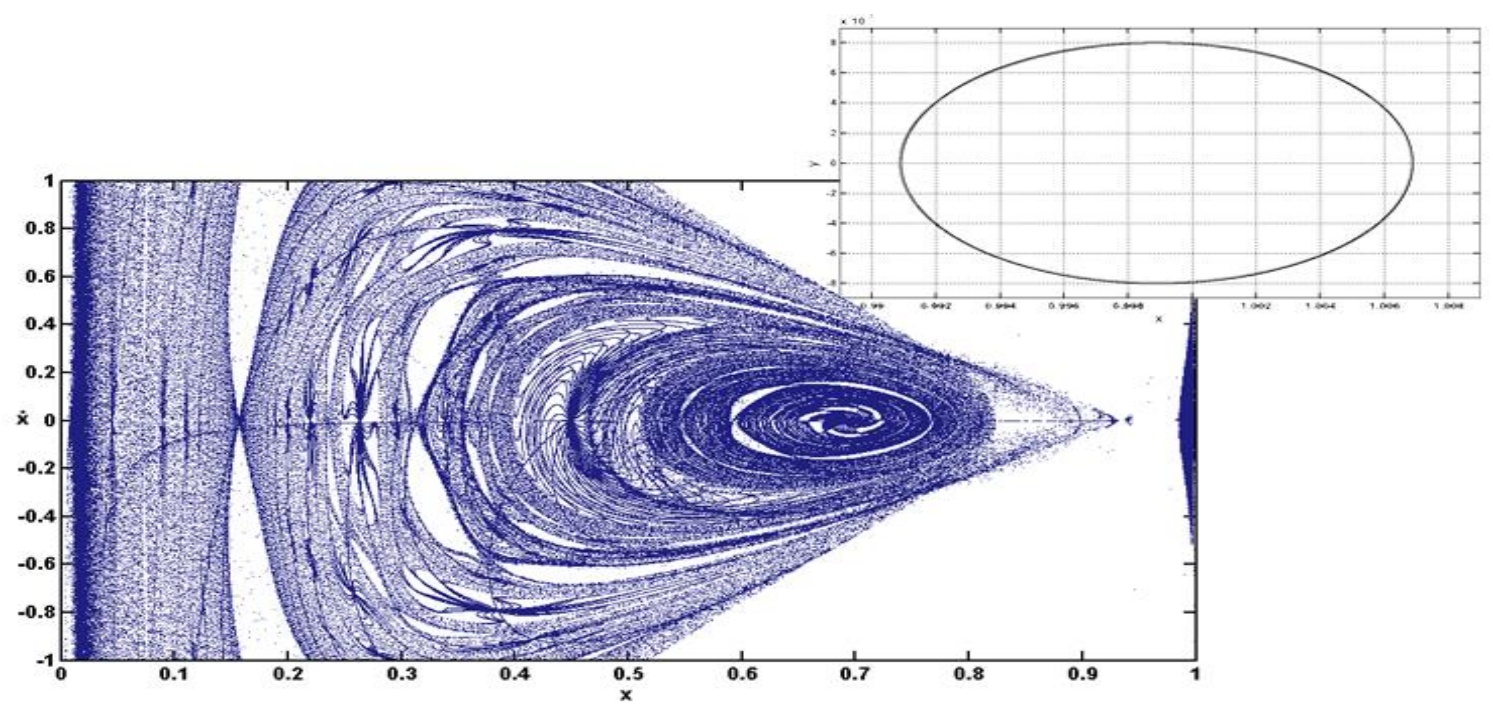

(C)

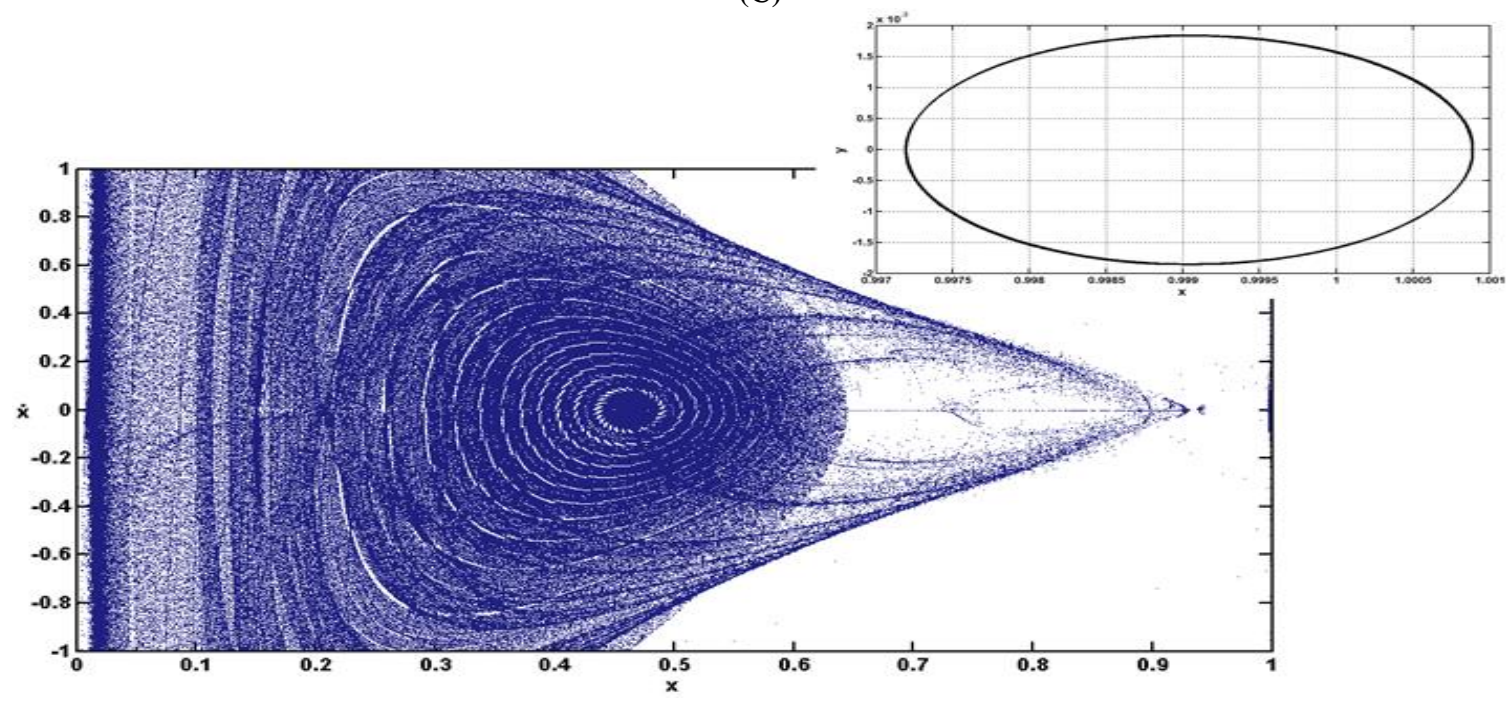

(D)

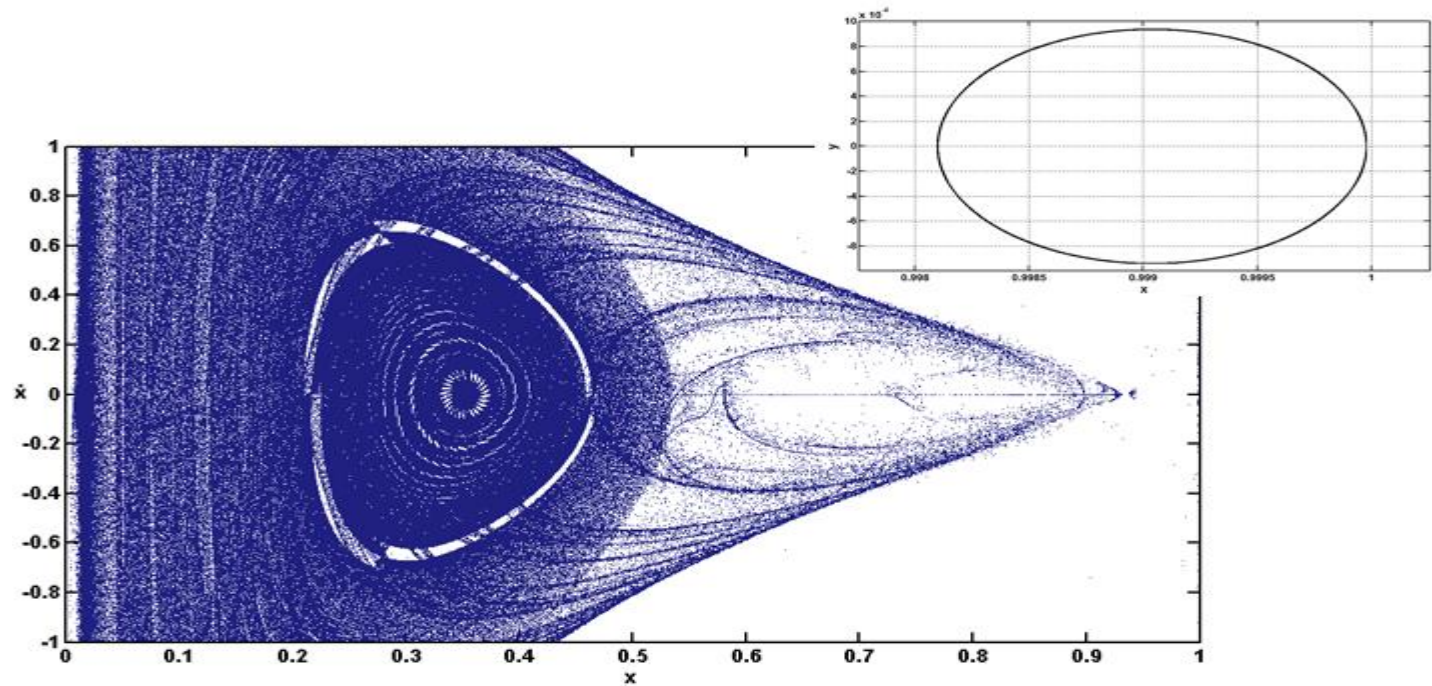

Fig. 1: Poincaré Surfaces of Section for Sun-Jupiter System with Various Values of $\mathrm{C}$ and Constant Value of $\mathrm{q}=1$ and Periodic Orbits around Jupiter: (A) $\mathrm{C}=3.0, \mathrm{X}=0.9609$; (B) $\mathrm{C}=3.1, \mathrm{X}=0.9914$; (C) $\mathrm{C}=3.5, \mathrm{X}=0.9972$; (D) $\mathrm{C}=4.0, \mathrm{X}=0.9981$.

It is found that the size of the periodic orbits around Jupiter decreases with the increase in $\mathrm{C}$ as shown in Fig. 2 and move towards Jupiter and collide with it at $\mathrm{C}=4$. Further, with the increase in the value of $\mathrm{C}$ to 4.2 , this orbit bifurcates into two orbits at locations $\mathrm{x}_{1}=0.9983$ and $\mathrm{x}_{2}=0.9998$; first one as retrograde orbit and the second one as direct orbit, as shown in Fig. 3. This shows that a new family of periodic orbit bifurcates from the original one which is contradictory to the properties of orbits. The direct periodic orbit belongs to the $g$ type family of periodic orbits and the bifurcated retrograde orbit belongs to the f type family of periodic orbits (Szebehely [25]).

In the present dynamical system, the KAM tori near the smaller primary is used to measure the degree of stability of the periodic orbits around Jupiter with respect to the region around it in the 
phase space. Fig. 4 provides the location of periodic orbit as a function of the Jacobi constant. These results are generated from the Poincaré surfaces of section, considering the size of the islands near Jupiter in the line of conjunction for each Jacobi constant $\mathrm{C}$. In Fig.4, the straight line corresponds to the leftmost tip and the dotted line corresponds to the rightmost tip of the island near Jupiter in the PSS of the Sun-Jupiter system. As seen from the figure, the degree of stability of the periodic orbits is seen to be maximum at around $\mathrm{C}=3.02$

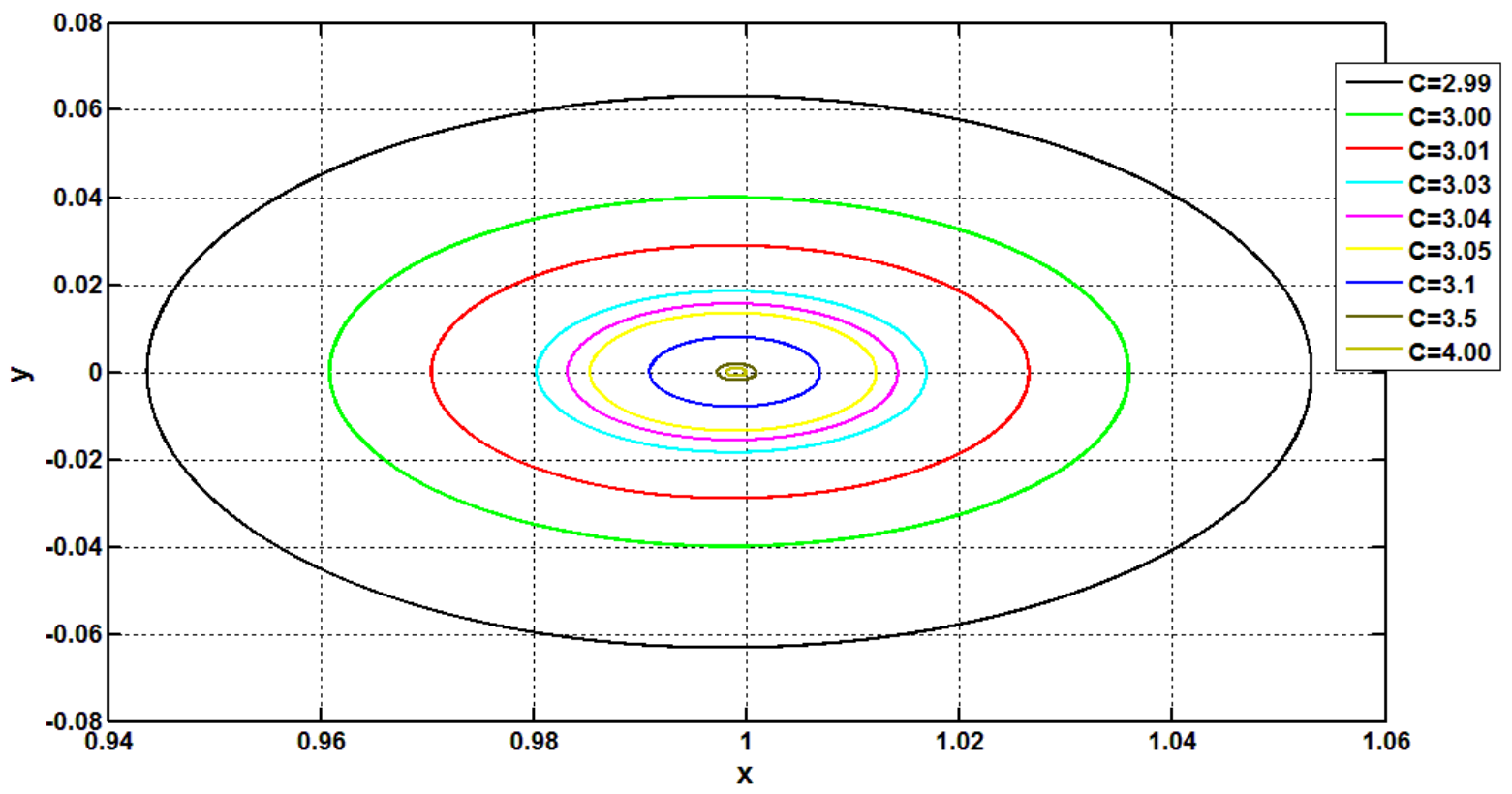

Fig. 2: Variation of Periodic Orbit around Jupiter for different values of $\mathrm{C}$ at $\mathrm{q}=1$.

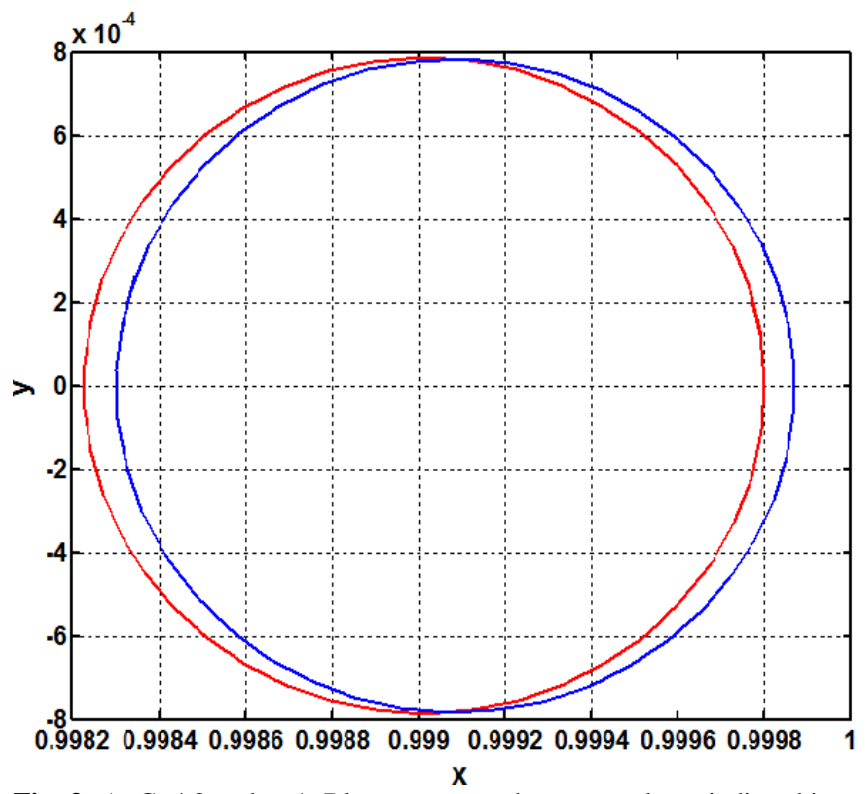

Fig. 3: At $\mathrm{C}=4.2$ and $\mathrm{q}=1$, Blue represents the retrograde periodic orbit a $\mathrm{X}_{1}=0.9983$ and Red represents the direct periodic orbit at $\mathrm{X}_{2}=0.9998$.

\section{Variation of bifurcated family of $f$ type pe- riodic orbits from $\mathrm{g}$ type periodic orbit for different values of $\mu$}

To study these orbits, we have generated PSS for one value of $\mathrm{C}$ at a time with $\mu$ varying from 0.01 to 0.5 , as shown in Figs. 5 a to $5 \mathrm{f}$.

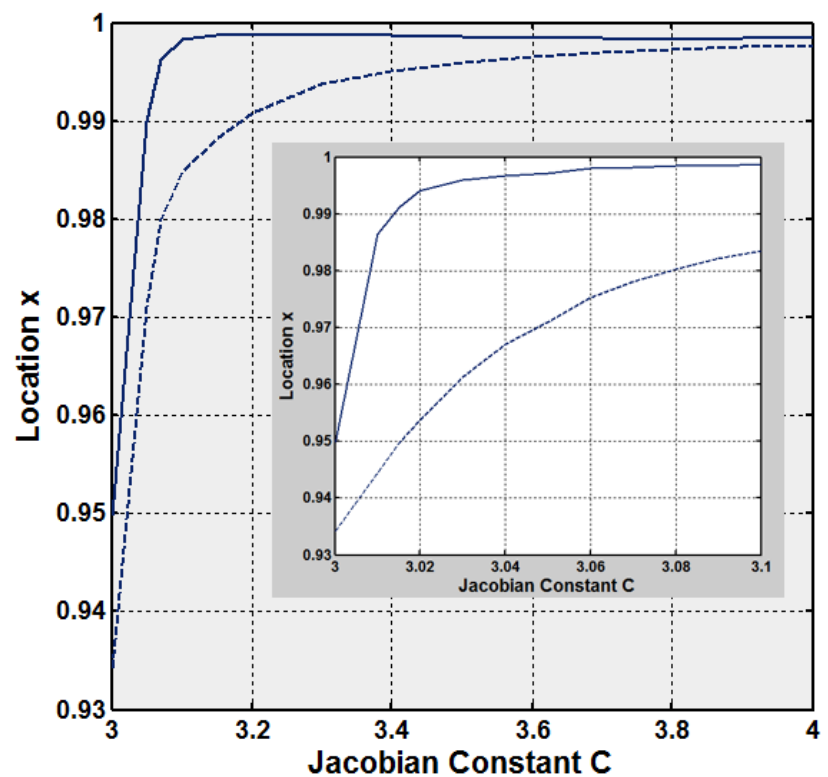

Fig. 4. Width of KAM Tori and Location of Periodic Orbits for the SunJupiter System at $\mathrm{q}=1$.

The periodic orbits for $0<\mu \leq 0.1$ are found to be elliptic orbits with eccentricity less than 0.2 , as shown in Figs. 5a to $5 \mathrm{~b}$ and those found for $\mu>0.1$ are elliptic orbits with eccentricity greater than 0.2, as shown in Figs. 5c to 5f. 
(A)

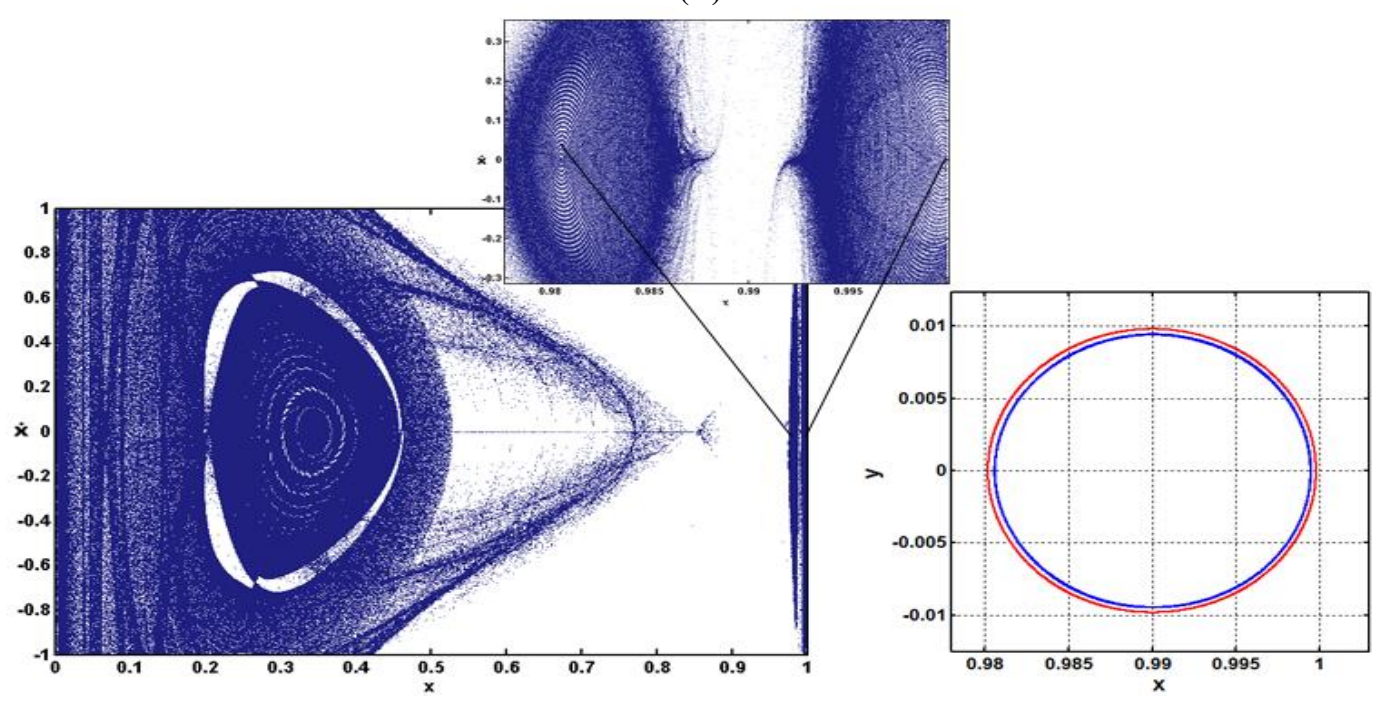

(B)

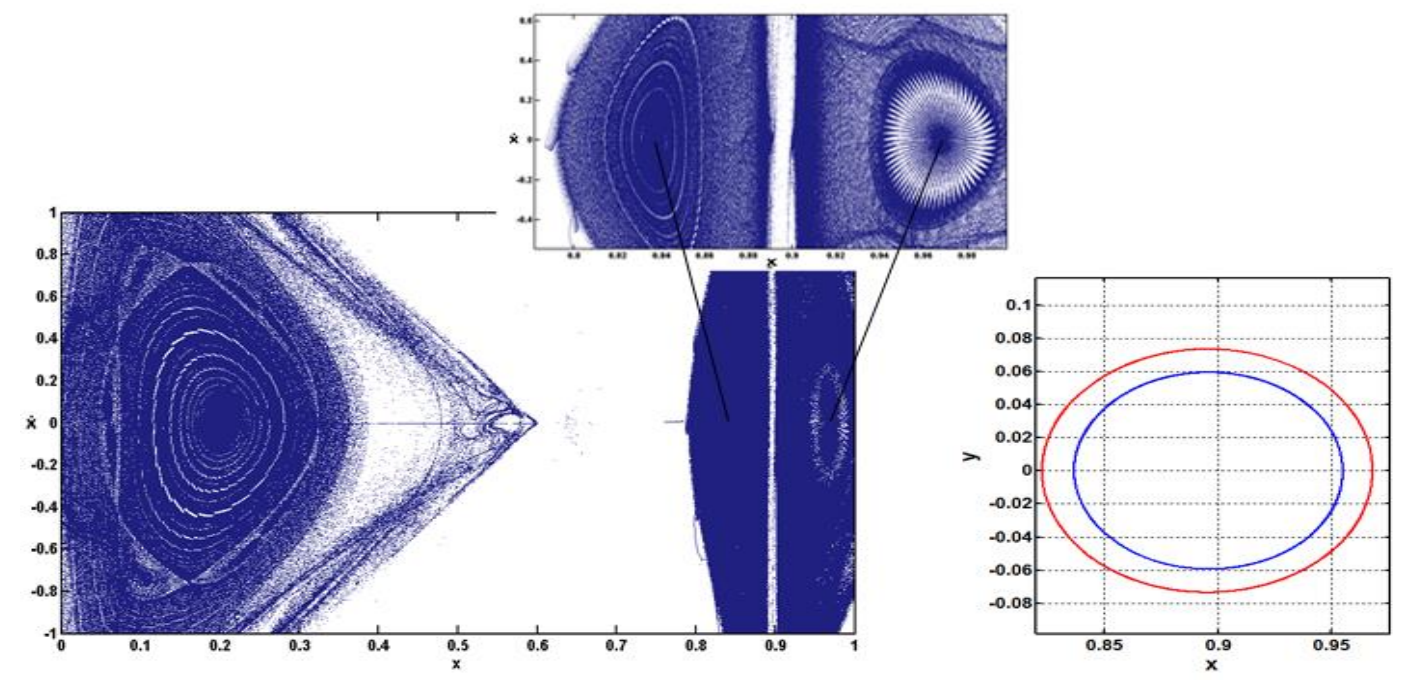

(C)

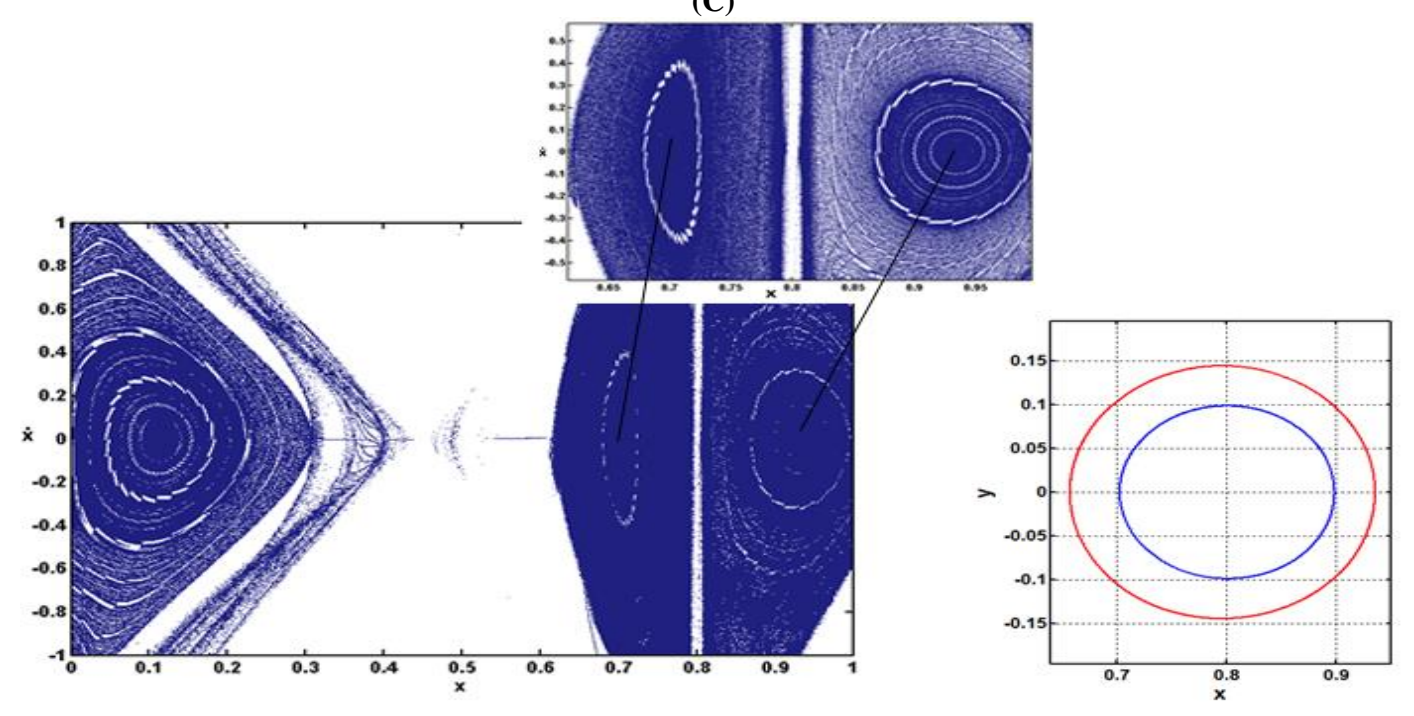




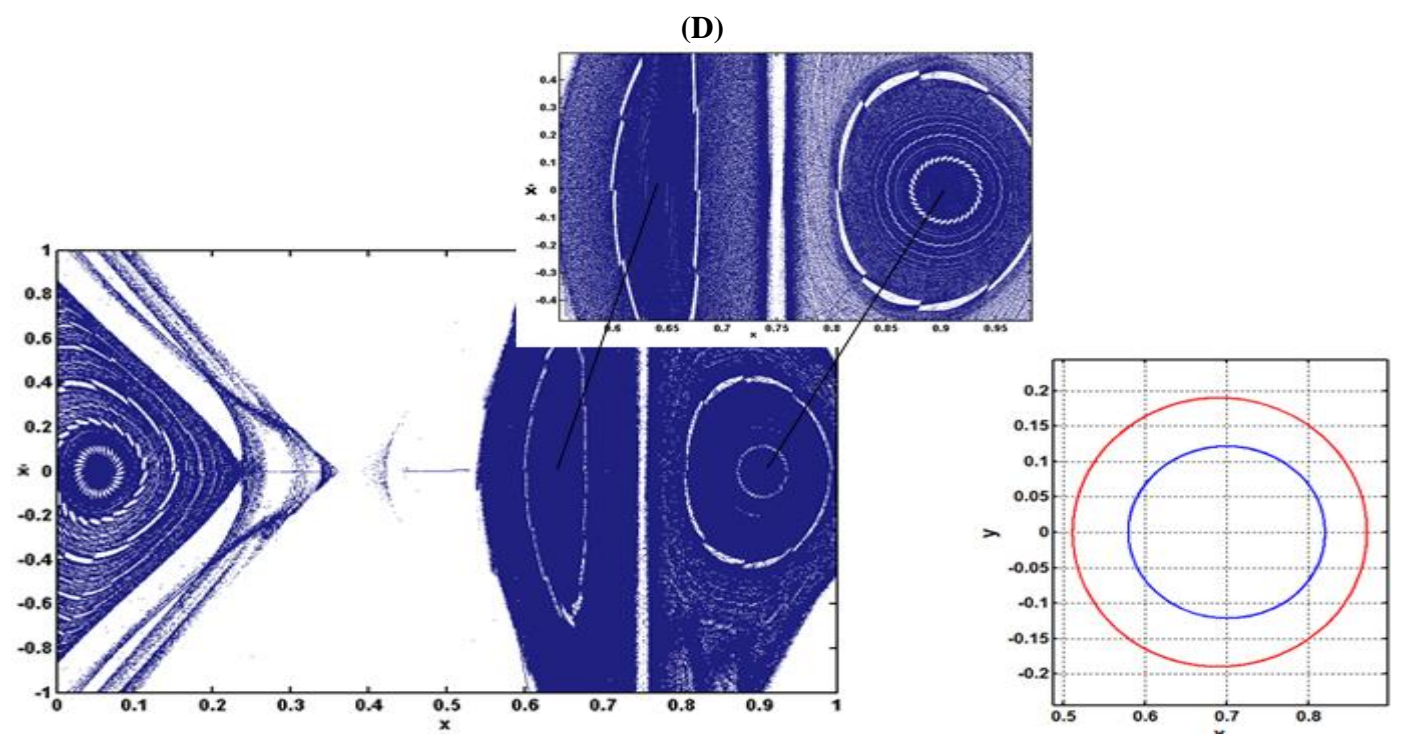

(E)

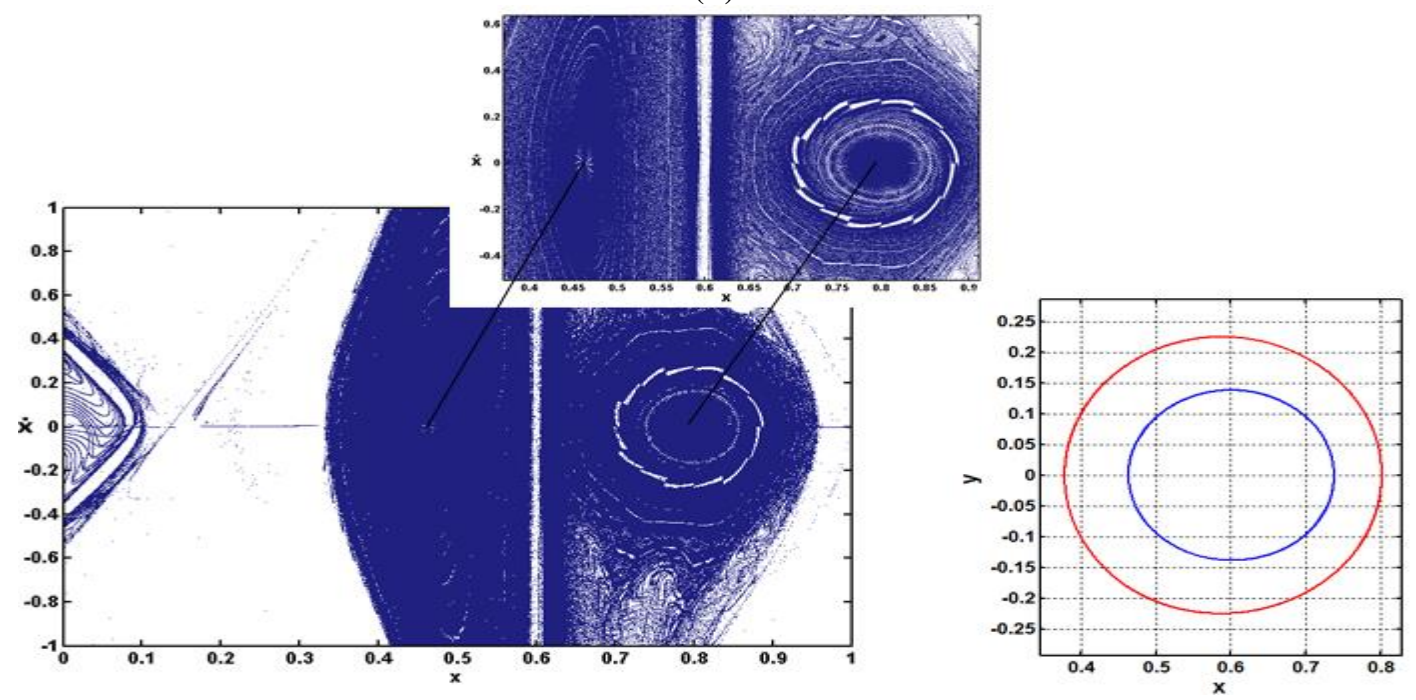

(F)

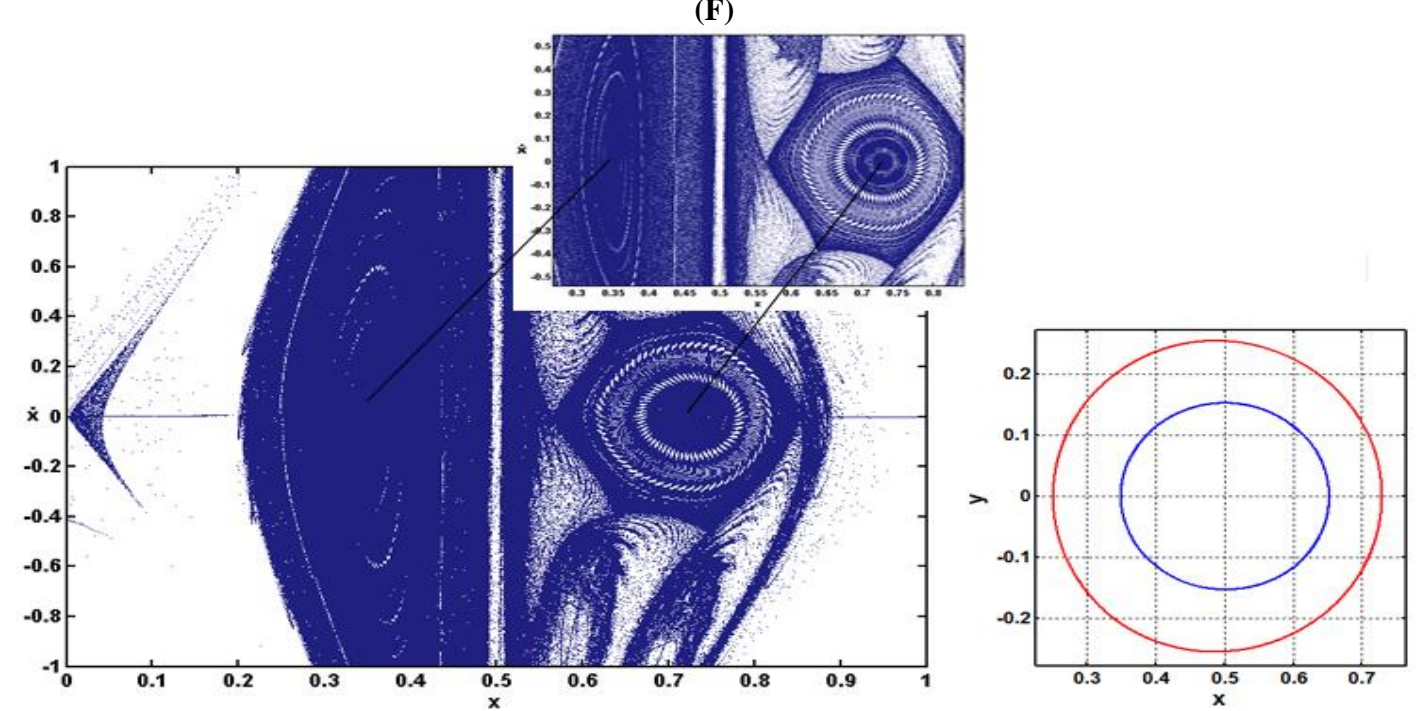

Fig. 5: Poincaré Surfaces of Section for Jacobi Constant $\mathrm{C}=4.2$, $\mathrm{q}=1$ and the Periodic Orbits (Red- Family G; Blue- Family F) for Different Mass Ratios: (A) $\mu=0.01$ and $X_{1}=0.9806, X_{2}=0.9998$; (B) $\mu=0.1$ and $X_{1}=0.8366, X_{2}=0.9681$; (C) $\mu=0.2$ and $X_{1}=0.7023, X_{2}=0.9356$; (D) $\mu=0.3$ and $X_{1}=0.5800$, $\mathrm{X}_{2}=0.8725$; (E) $\mu=0.4$ and $\mathrm{X}_{1}=0.4631, \mathrm{X}_{2}=0.8022$; (F) $\mu=0.5$ and $\mathrm{X}_{1}=0.3492, \mathrm{X}_{2}=0.7291$. 


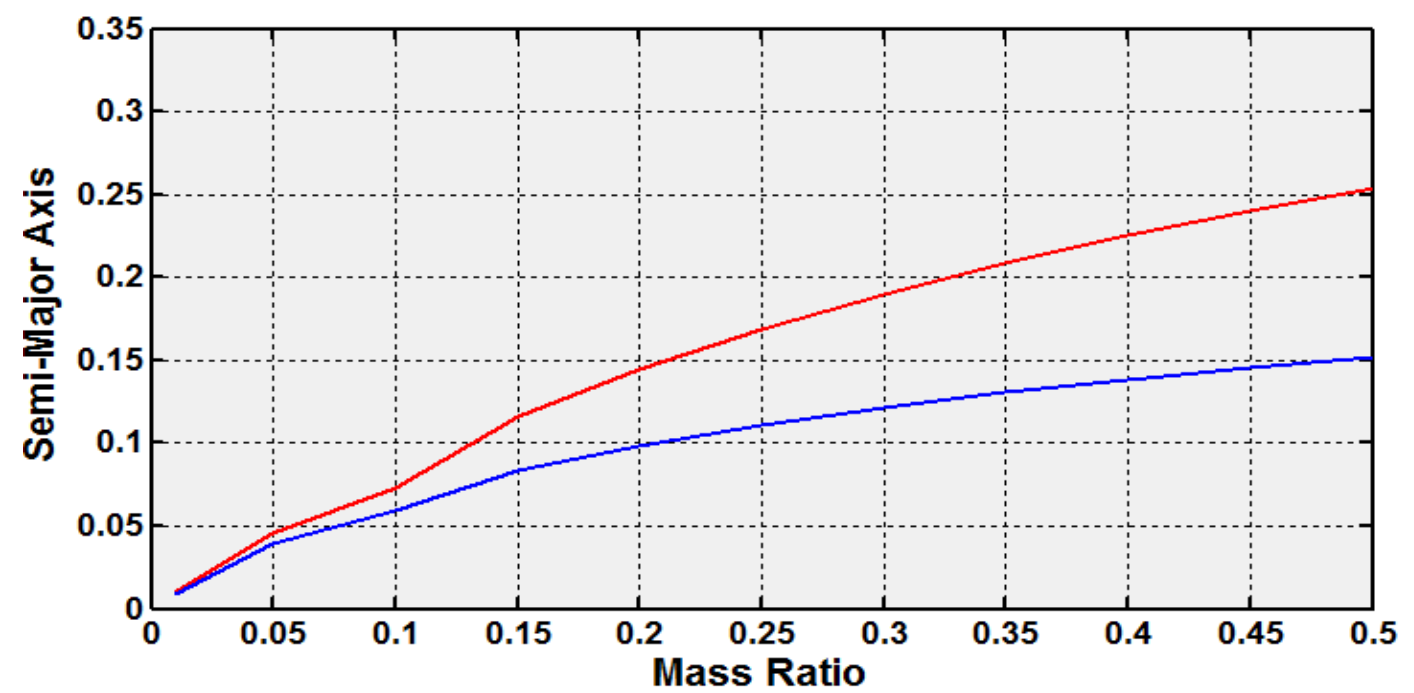

Fig. 6a: Variation of Semi-Major Axis of new Periodic Orbits with mass ratio when $q=1$.

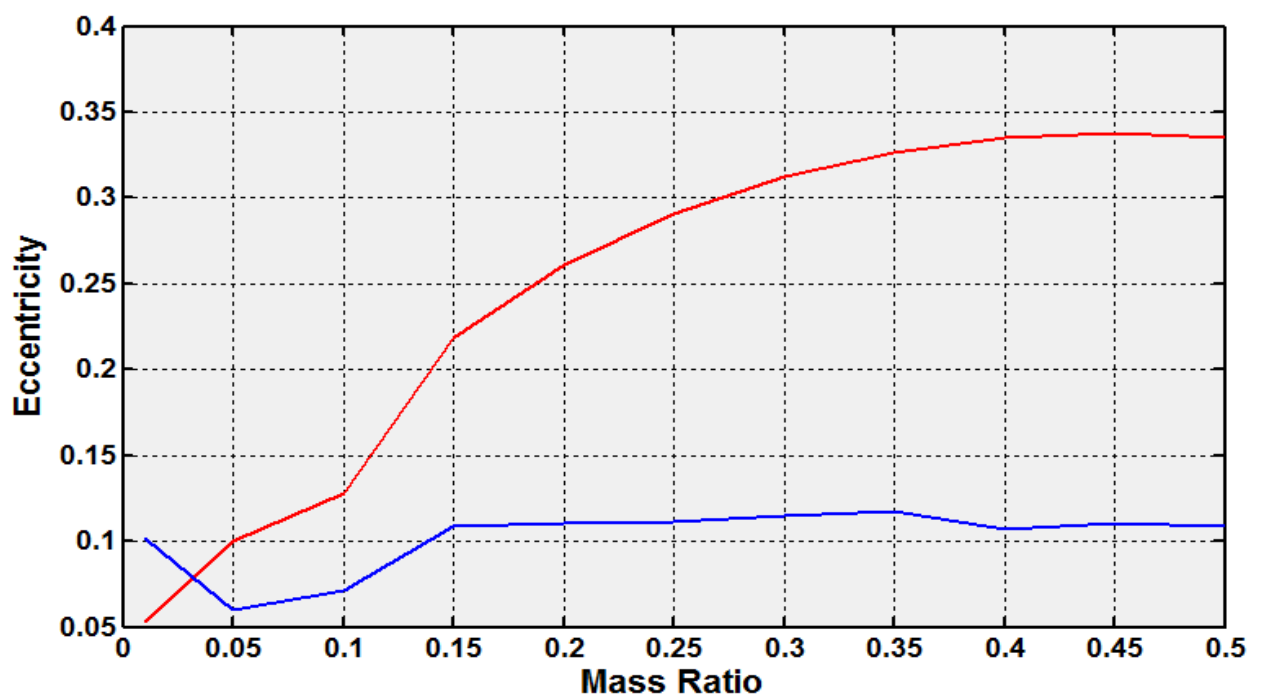

Fig. 6b: Variation of Eccentricities of new Periodic Orbits with mass ratio when $q=1$.

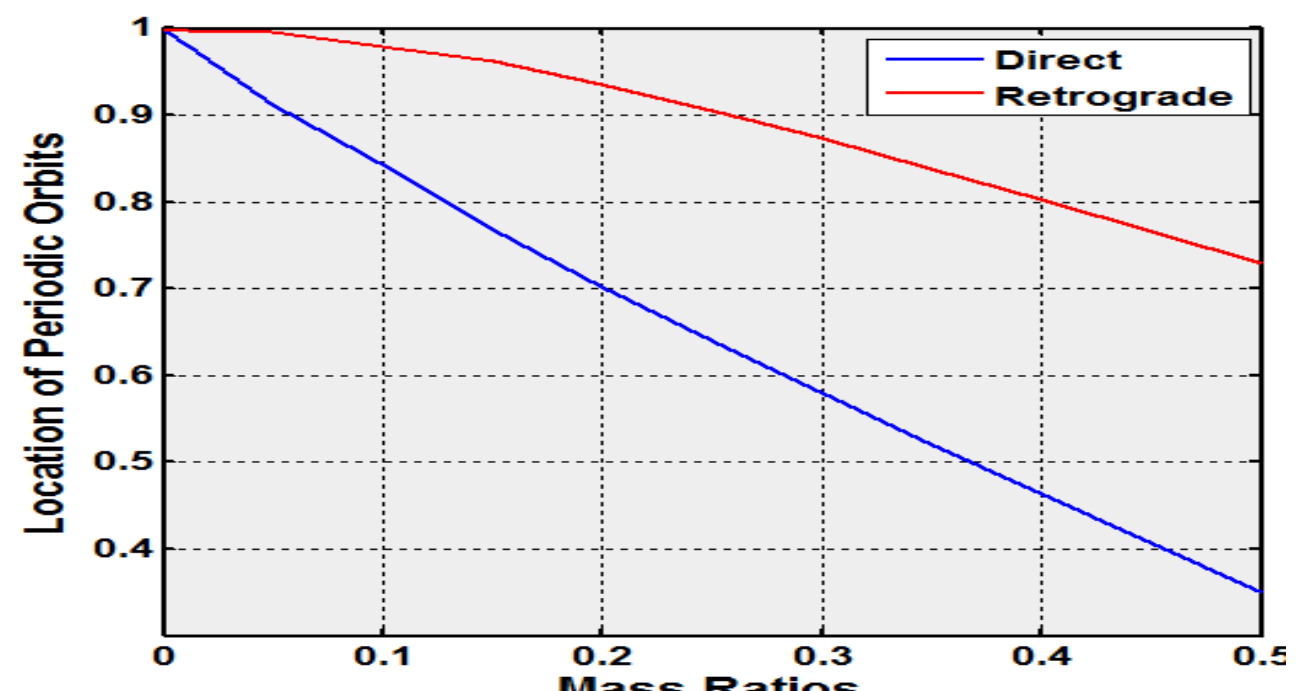

Fig. 7: Location of the new Periodic Orbits with various mass ratio $\mu$ up to 0.5 when $q=1$.

The size and eccentricity of these periodic orbits increases with increase in $\mu$, as shown in figures $6 \mathrm{a}, 6 \mathrm{~b}$.

Fig. 7 provides the locations of the new periodic orbits for $\mu$ up to 0.5 for constant Jacobi constant $\mathrm{C}$ and radiation pressure q. It is noted that, family $\mathrm{f}$ as well as family $\mathrm{g}$ periodic orbits and the distance between them increases with increase in $\mu$.
6. Effect of solar radiation pressure on semimajor axis and eccentricity on the bifurcated families of periodic orbits

To study the effect of solar radiation pressure on the orbital parameters of the new orbits, the more massive primary is considered as a source of radiation. Graphs have been plotted for varia- 
tion of semi-major axis with different values of $\mu$ and the effect of radiation pressure is also represented. Figs. 10 and 11 show the effect of radiation pressure on the semi-major axis of periodic orbits with mass ratio variation. It is seen that the effect of radiation pressure decreases the size of semi-major axis for both fami- lies of periodic orbits for different values of $\mu$. The variation of eccentricity and semi-major axis of both: retrograde and direct orbits with radiation pressure effects have been computed and are shown in Tables 1-4.

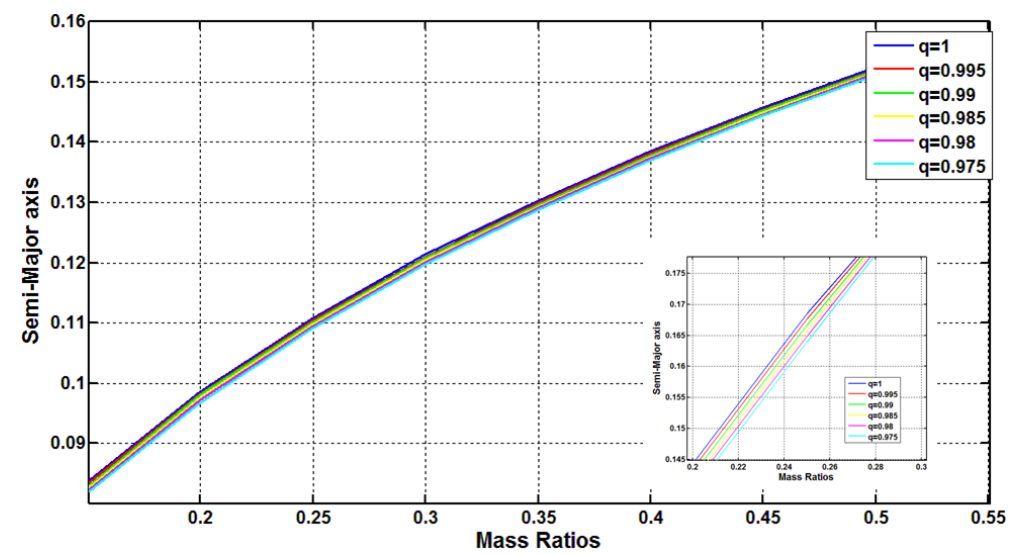

Fig. 10: Effect of Radiation Pressure on Semi-Major Axis of Retrograde Orbit for varying $\mu$.

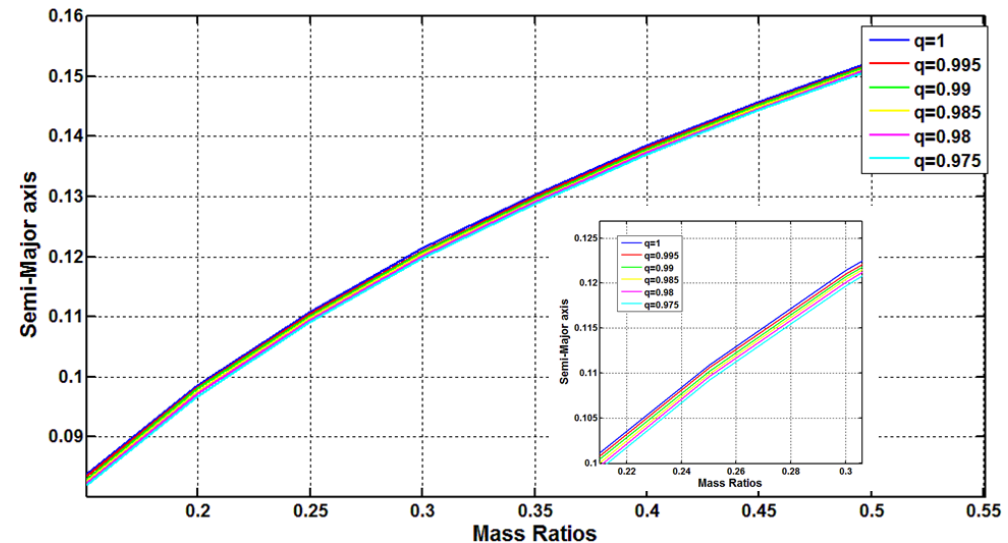

Fig. 11: Effect of Radiation Pressure on Semi-Major Axis of Direct Orbit for varying $\mu$.

Table 1: Semi-Major Axis of Retrograde Orbit for Various $\mu$ with Radiation Pressure

\begin{tabular}{llllll}
\hline$\mu$ & 1 & 0.995 & 0.99 & 0.985 & 0.98 \\
\hline 0.000953728 & 0.0008 & 0.0008 & 0.0008 & 0.0008 & 0.000764 \\
0.012131429 & 0.00955 & 0.009469 & 0.00942 & 0.009332 & 0.009263 \\
0.05 & 0.03898 & 0.03872 & 0.03841 & 0.03813 & 0.03785 \\
0.104323953 & 0.05934 & 0.05899 & 0.05865 & 0.05831 & 0.05805 \\
0.15 & 0.08377 & 0.0834 & 0.08302 & 0.08265 & 0.08236 \\
0.2 & 0.0987 & 0.09833 & 0.09804 & 0.09759 & 0.09723 \\
0.25 & 0.1109 & 0.1106 & 0.1102 & 0.1099 & 0.1096 \\
0.3 & 0.1214 & 0.121 & 0.1207 & 0.1204 & 0.1201 \\
0.35 & 0.1304 & 0.13 & 0.1297 & 0.1294 & 0.1291 \\
0.4 & 0.1385 & 0.1382 & 0.1379 & 0.1376 & 0.1373 \\
0.45 & 0.1457 & 0.1454 & 0.1452 & 0.1449 & 0.1095 \\
0.5 & 0.1524 & 0.1522 & 0.1519 & 0.1517 & 0.1445 \\
\end{tabular}

Table 2: Eccentricity of Retrograde Orbit for Various $\mu$ with Radiation Pressure

\begin{tabular}{llllll}
\hline \multicolumn{5}{c}{ Table 2: Eccentricity of Retrograde Orbit for Various $\mu$ with Radiation Pressure } \\
\hline$\mu$ & 1 & 0.995 & 0.99 & 0.985 & 0.98 \\
0.000953728 & 0.164512 & 0.202655 & 0.238062 & 0.268606 & 0.975 \\
0.012131429 & 0.052159 & 0.063317 & 0.065129 & 0.082743 & 0.298661 \\
0.05 & 0.06403 & 0.078669 & 0.055873 & 0.03966 & 0.052961 \\
0.104323953 & 0.068651 & 0.055218 & 0.041283 & 0.018519 & 0.051383 \\
0.15 & 0.105786 & 0.103741 & 0.100461 & 0.10421 & 0.097338 \\
0.2 & 0.110096 & 0.105636 & 0.118433 & 0.109785 & 0.111114 \\
0.25 & 0.112179 & 0.112331 & 0.108452 & 0.112687 & 0.109064 \\
0.3 & 0.118128 & 0.111168 & 0.114943 & 0.115086 & 0.11284 \\
0.35 & 0.113992 & 0.107262 & 0.103754 & 0.103874 & 0.11523 \\
0.4 & 0.113816 & 0.113939 & 0.110859 & 0.107676 & 0.103994 \\
0.45 & 0.107859 & 0.101438 & 0.104828 & 0.101613 & 0.049853 \\
0.5 & 0.102328 & 0.105538 & 0.09925 & 0.099315 & 0.098277 \\
\hline
\end{tabular}


Table 3: Semi-Major Axis of Direct Orbits for Various $\mu$ with Radiation Pressure

\begin{tabular}{|c|c|c|c|c|c|c|}
\hline$\mu \quad q \rightarrow$ & 1 & 0.995 & 0.99 & 0.985 & 0.98 & 0.975 \\
\hline 0.000953728 & 0.0008 & 0.0008 & 0.0008 & 0.000774 & 0.000767 & 0.000761 \\
\hline 0.012131429 & 0.009875 & 0.0098 & 0.009717 & 0.00965 & 0.009571 & 0.009484 \\
\hline 0.05 & 0.04553 & 0.04512 & 0.04472 & 0.04431 & 0.04391 & 0.04351 \\
\hline 0.104323953 & 0.07338 & 0.0798 & 0.07258 & 0.07218 & 0.07174 & 0.07134 \\
\hline 0.15 & 0.1162 & 0.1157 & 0.1152 & 0.1147 & 0.1141 & 0.1135 \\
\hline 0.2 & 0.1443 & 0.1434 & 0.1425 & 0.1416 & 0.1407 & 0.1398 \\
\hline 0.25 & 0.1686 & 0.1677 & 0.1667 & 0.1658 & 0.1649 & 0.164 \\
\hline 0.3 & 0.1898 & 0.1894 & 0.189 & 0.1886 & 0.1881 & 0.1875 \\
\hline 0.35 & 0.2085 & 0.2076 & 0.2068 & 0.2059 & 0.2051 & 0.2042 \\
\hline 0.4 & 0.2252 & 0.2244 & 0.2236 & 0.2228 & 0.222 & 0.2211 \\
\hline 0.45 & 0.2404 & 0.2396 & 0.2388 & 0.2381 & 0.2373 & 0.2365 \\
\hline 0.5 & 0.2541 & 0.2534 & 0.2527 & 0.252 & 0.2513 & 0.2506 \\
\hline
\end{tabular}

Table 4: Eccentricity of Direct Orbit for Various $\mu$ with Radiation Pressure

\begin{tabular}{|c|c|c|c|c|c|c|}
\hline $\mathrm{q} \rightarrow$ & 1 & 0.995 & 0.99 & 0.985 & 0.98 & 0.975 \\
\hline 0.000953728 & 0.137513 & 0.184939 & 0.223299 & 0.244622 & 0.209968 & 0.166369 \\
\hline 0.012131429 & 0.071112 & 0.020202 & 0.059127 & 0.02879 & 0.066208 & 0.058014 \\
\hline 0.05 & 0.088833 & 0.086725 & 0.087111 & 0.084905 & 0.070739 & 0.071063 \\
\hline 0.104323953 & 0.12548 & 0.136192 & 0.14147 & 0.151214 & 0.15258 & 0.157465 \\
\hline 0.15 & 0.212346 & 0.226243 & 0.235855 & 0.246866 & 0.252594 & 0.253246 \\
\hline 0.2 & 0.259673 & 0.26047 & 0.259979 & 0.259479 & 0.257642 & 0.255767 \\
\hline 0.25 & 0.292041 & 0.291831 & 0.290718 & 0.290497 & 0.290274 & 0.290047 \\
\hline 0.3 & 0.313321 & 0.320638 & 0.032867 & 0.335779 & 0.341379 & 0.344826 \\
\hline 0.4 & 0.334218 & 0.335422 & 0.336004 & 0.33596 & 0.032562 & 0.335311 \\
\hline 0.45 & 0.339174 & 0.339144 & 0.339114 & 0.339597 & 0.339567 & 0.338953 \\
\hline 0.5 & 0.337339 & 0.337851 & 0.337753 & 0.338208 & 0.331109 & 0.338569 \\
\hline
\end{tabular}

\section{Conclusion}

Using Poincaré surfaces of section method, we have studied the presence of a bifurcated family $f$ periodic orbits from family $g$ periodic orbits for various mass ratios in the photogravitational circular restricted three-body problem. We have determined the nature, stability and size of these orbits depending on the Jacobian constant. These periodic orbits belong to the adjacent separate families of periodic orbits: family $g$ and family $f$ orbits. As the Jacobian constant increases, these orbits gradually move away from the smaller primary. We study the evolution of these bifurcated f family periodic orbits that are around the smaller primary by increasing the value of Jacobi constant. This study shows that increase in $\mu$ increases the size of these periodic orbits for some values of Jacobian constant. These families of periodic orbits found within $0<\mu \leq 0.1$ have eccentricity less than 0.2 and those found for $\mu>0.1$ are elliptic orbits with eccentricity greater than 0.2 . These orbits are found in the three systems: Pluto-Charon $(\mu=0.104323953)$, Earth-Moon $(\mu=0.012131429)$ and Sun-Jupiter $(\mu=0.000953728)$ in the solar system when $C>4$ without considering any perturbation effects. With inclusion of solar radiation pressure, the deviations in the parameters: semi-major axis, eccentricity and time period of these families of periodic orbits are found.

\section{References}

[1] Beevi, A.S. \& Sharma, R.K. (2011) Analysis of periodic orbits in the Saturn-Titan system using the method of Poincare section surfaces. Astrophysics and Space Science 333, 37-48. https://doi.org/10.1007/s10509-011-0630-0.

[2] Beevi, A.S. \& Sharma, R.K. (2012) Oblateness effect of Saturn on periodic orbits in Saturn- Titan restricted three-body problem. Astrophysics and Space Science 340, 245-261. https://doi.org/10.1007/s10509-012-1052-3.

[3] Broucke, R.A. (1968) Periodic orbits in the restricted three-body problem with Earth-Moon masses. Pasadena, CA: Jet Propulsion Lab.

[4] Bruno, A. (1990) The Restricted 3-Body Problem. Plane Periodic Orbits. Nauka, 296.

[5] Bruno, A. \& Varin, V. (2006) On families of periodic solutions of the restricted three-body problem. Celestial Mechanics \& Dynamical Astron. 95, 27-54. https://doi.org/10.1007/s10569-006-9021-1.
[6] Dutt, P. \& Sharma, R.K. (2010) Analysis of periodic and quasiperiodic orbits in the Earth- Moon system. Journal of Guidance, Control and Dyn. 33, 1010-1017. https://doi.org/10.2514/1.46400.

[7] Dutt, P. \& Sharma, R.K. (2011) Evolution of periodic orbits near the Lagrangian point L2. Advances in Space Res. 47, 1894-1904. https://doi.org/10.1016/j.asr.2011.01.024.

[8] Dutt, P. \& Sharma, R.K. (2011) Evolution of Periodic Orbits in the Sun-Mars system. Journal of Guidance, Control and Dyn. 34, 635 644. https://doi.org/10.2514/1.51101.

[9] Hénon, M. (1969) Numerical exploration of the restricted problem. V.Astron.Astrophys. 1, 223-238.

[10] Jefferys, W. H. (1971) An Atlas of Surface of Section for the Restricted Problem of Three bodies. Austin,Texas: Dept. of Astronomy,University of Texas.

[11] Kalvouridis, T., Arribas, M. \& Elipe, A. (2007) Parametric Evolution of Periodic Orbits in Restricted Four-Body Problem with Radiation Pressure. Planetary and Space Sci. 55, 475-493. https://doi.org/10.1016/j.pss.2006.07.005.

[12] Murray, C.D. \& Dermott, S.F. (1999) Solar System Dynamics. Cambridge: Cambridge University Press.

[13] Poincaré, H. (1892) Les Methodes Nouvellas de la Mechanique Celeste (Vol. 1). Paris: Gauthier-Villars.

[14] Poynting, J. (1904) Radiation in the Solar system: It's Effect on Temperature and its pressure on Small Bodies. Philosophical Transactions of the Royal Society of London.Series A, Physical Sciences and Engineering 202, 525-552. https://doi.org/10.1098/rsta.1904.0012.

[15] Pushparaj, N. and Sharma, R.K. (2017) Interior Resonance Periodic Orbits in the Photogravitational Restricted Three-Body Problem. Advances in Astrophys. 2, 25-34.

[16] Radzievskii, V. (1950) The Restricted problem of Three Bodies Taking Account of Light Pressure. Akademii Nauk USSR, Astronomicheskii Zhurnal 27, 250-256.

[17] Robertson, H. (1937) Dynamical Effects of Radiation in the solar system. Monthly Noties of the Royal Astronomical Society 97, 423-438. https://doi.org/10.1093/mnras/97.6.423.

[18] Sharma, R.K. (1975) Perturbations of Lagrangian points in the restricted three-body problem, Indian J Pure and Appl. Math. 6, 1099. 1102.

[19] Sharma, R.K. ( 1982) On linear stability of triangular libration points of the photogravitational three-body problem when the more massive primary is an oblate spheroid, Sun and Planetary System, W. Fricke and G. Teleki (Eds.), D. Reidel Publishing Co., Dordrecht, Holland, 435-436.

[20] Sharma, R.K. (1987). The linear stability of libration points of the photogravitational restricted three-body problem when the smaller primary is an oblate spheroid, Astrophys. And Space Sci. 135, 271281. https://doi.org/10.1007/BF00641562. 
[21] Sharma, R. K. \& Subba Rao, P.V. (1976) Stationary solutions and their characteristic exponents in the restricted three-body problem when the more massive primary is an oblate spheroid. Celestial Mech. 13, 137-149. https://doi.org/10.1007/BF01232721.

[22] Sharma, R. K. \& Subba Rao, P.V. (1986) on finite periodic orbits around the equilateral solutions of the planar restricted three-body problem, International workshop on Space Dynamics and Celestia mechanics, November 1985, Delhi, India, K. B. Bhatnagar (Ed), D. Reidel Publishing Co., Dordrecht, Holland, pages 71-85. https://doi.org/10.1007/978-94-009-4732-0 8.

[23] Smith, R. H., \& Szebehely, V. (1993) the onset of chaotic motion in the restricted problem of three bodies. Celest. Mech. 56, 409-425. https://doi.org/10.1007/BF00691811.

[24] Subba Rao, P. V. \& Sharma R. K. (1988) Oblateness effect on finite periodic orbits at L4, IAF-88-300, 5 pages, 39th Congress of the International Astronautical Federation, October 1988, Bangalore, India.

[25] Szebehely, V. (1967) Theory of Orbits. San Diego: Academic Press.

[26] Voyatzis, G., Kotoulas, T. \& Hadjidemetriou, J. D. (2005) Symmetric and nonsymmetric periodic orbits in the exterior mean motion resonances with Neptune. Celestial Mechanics and Dynamical Astronomy, 91, 191-202. https://doi.org/10.1007/s10569-004-0891-9.

[27] Winter, O. C. \& Murray, C. D. (1994b) Atlas of the Planar, Circular, Restricted Threebody Problem: External orbits. Queen Mary and Westfield College,London: QMW Maths Notes.

[28] Winter, O. C. \& Murray, C. D. (1994a) Atlas of the Planar, Circular, Restricted Threebody Problem: Internal orbits. Queen Mary and Westfield College, London: QMW Maths Notes.

[29] Winter, O. C. \& Murray, C. D. (1997a) Resonance and chaos. I. First-order interior resonances. Astronomy and Astrophys. 319, 290-304.

[30] Winter, O. C. \& Murray, C. D. (1997b). Resonance and chaos. II. Exterior resonances and asymmetric libration. Astronomy and Astrophys. 328, 399-408.

[31] Zotos, E. E. (2015) Crash test for the Copenhagen problem with oblateness. Celest Mech \& Dyn Astron. 122, 75-99. https://doi.org/10.1007/s10569-015-9611-x. 